educational attainment and low sense of well-being. The impact of household food insecurity on an individual's quality of diet depends on their role within the household and mothers may be more adversely affected than other family members. Studies have linked food insecurity with mental health problems in women of childbearing age. This study examines the relationship between food insecurity, well-being and quality of diet in women of childbearing age.

Design Cross-sectional survey of educational attainment, food insecurity, well-being and quality of diet in women of childbearing age.

Setting Sure Start Children's Centres (SSCCs) in three south coast towns with high levels of disadvantage- Southampton, Gosport and Havant.

Participants 1022 women attending SSCCs between January and April 2009.

Main outcome measures Food insecurity, assessed using the short form of Blumberg household food security scale; dietary quality score from a 20-item food frequency questionnaire; well-being by the WHO-5 questionnaire.

Results $11 \%$ of women had insufficient money to buy food, and $9 \%$ reported going hungry. Women of lower educational attainment were twice as likely to be food insecure as women of higher educational attainment (prevalence rate ratio 2.02, 95\% CIs 1.42 to 2.86). Women who reported lower well-being were three times as likely to go hungry as women of higher well-being (prevalence rate ratio $3.18,95 \%$ CIs 2.04 to 4.93 .) Women who experienced food insecurity had poorer quality diets: becoming food insecure was accompanied by a 0.79 standard deviation reduction in dietary quality score $(95 \% \mathrm{CIs}-1.10$ to -0.48$)$. In a multivariate regression analysis, having lower educational attainment, lower well-being, being food insecure and going hungry were significant independent predictors of dietary quality, after adjusting for age and number of children in the household. Being food insecure predicted a 0.32 standard deviation reduction in dietary quality score $(95 \%$ CIs -0.61 to -0.28$)$, and going hungry a 0.45 standard deviation reduction (95\% CIs -0.77 to -0.12 ).

Conclusion Food insecurity was common: one in five women lived in food insecure households. Women of lower educational attainment were twice as likely to be food insecure as women of higher educational attainment. National survey data indicate that disadvantaged young women have diets of poor quality. Our study suggests that food insecurity and low sense of well-being are aspects of disadvantage that predict poor quality of diet.

\section{SYSTEMATIC REVIEW AND META-ANALYSIS OF SCHOOL- BASED INTERVENTIONS TO IMPROVE FRUIT AND VEGETABLE INTAKE}

doi:10.1136/jech.2010.120956.68

${ }^{1} \mathrm{C}$ E L Evans, ${ }^{2} \mathrm{D}$ C Greenwood, ${ }^{1} \mathrm{~J}$ E Cade. ${ }^{1}$ Nutritional Epidemiology group, University of Leeds, Leeds, UK; ${ }^{2}$ Biostatistics Unit, University of Leeds, Leeds, UK

Objective The aims of the review were to identify school-based randomised and non-randomised controlled trials to increase daily or lunchtime fruit and vegetable intake in children and to determine the impact of school-based interventions to change fruit and vegetable consumption at lunchtime and over the whole day.

Design A systematic literature review was carried out to identify appropriate trials. This was followed by meta-analysis techniques to determine the pooled estimate of the difference in daily fruit and vegetable intake in the intervention group compared with the control group.

Participants Trials carried out in schools where children were aged 5 to 11 years were included. All trials reported in English language journals were eligible.
Results 28 randomised and non-randomised controlled trials were identified that reported daily fruit and/or vegetable intake. A median intake of 0.4 portions more fruit and vegetables was consumed in the intervention group compared to the control group. The qualitative review of 7 studies reporting lunchtime intake, either in addition to daily intake or independently in studies concentrating solely on lunchtime intake, revealed a median difference of 0.2 portions more fruit and vegetables in the intervention group at lunchtime. The metaanalysis of daily intake included 13 studies classified into one of two groups: behavioural change studies with a school and/or home component that relied on families improving eating behaviour; and free school fruit and vegetable scheme where fruit and vegetables are distributed to children. The short term impact of both type of programme was determined using the follow up data collected within 3 months of the end of the intervention. This was the longest followup period in most cases. The pooled estimates $(95 \% \mathrm{CI}$ ) for behavioural change studies and free fruit and vegetable schemes were 0.43 (0.21 to 0.65$)$ and 0.44 (0.20 to 0.67$)$ portions respectively. The pooled estimate $(95 \% \mathrm{CI})$ for all studies was 0.42 (95\% CI 0.27 to 0.58$)$ portions more in the intervention group. The majority of the difference was due to fruit not vegetables. Heterogeneity was high for the meta-analysis with lunchtime intake but reasonable for daily intake. Conclusion School-based interventions have the potential to moderately improve fruit and vegetable intake in children, with approximately half of the increase attributable to improvements in lunchtime intake.

\section{Physical activity \\ 069 LONGITUDINAL ASSOCIATION BETWEEN EARLY LIFE ADVERSITY AND PHYSICAL ACTIVITY AT AGE 36 YEARS}

doi:10.1136/jech.2010.120956.69

D Gaysina, R Cooper, D Kuh, G Mishra. MRC Unit for Lifelong Health and Ageing, University College London, London, UK

Background Periods early in life, between birth and adolescence, could be especially important for the establishment of health behaviours. Early adversity may influence the level and types of physical activity in adulthood.

Objectives To investigate the associations between early adversity and adult physical activity, and the role of depression and education as potential mediators of these associations. Structural equation models were used to 1) identify five latent factors of early adversity: socio-economic conditions (father's unskilled job, lack of home amenities and overcrowding), parental health (parental poor heath, maternal neuroticism); family structure during childhood (death of parents, divorce), chronic illness (hospitalisation for $>1$ month); and social isolation; 2 ) model the effect of depression/anxiety (at ages 15 and 36 years) and education level (at age 26 year) on the relationships between early adversity and physical activity.

Design Prospective cohort study.

Setting England, Scotland and Wales.

Participants Approximately 3300 men and women from the MRC National Survey of Health and Development, followed up since birth in March 1946.

Main outcome measures Four physical activity types measured at age 36 years-cycling/walking, heavy gardening, sports/recreational activities, and activity during the working day-with three levels of intensity (most active, less active, inactive)

Results There was no evidence of direct paths between early adversity and adult physical activity $(p>0.05)$. However, there was a strong indirect effect of socio-economic adversity on activity at work (0.24) and on sports/recreational activity $(-0.10)$ via education. The significant indirect effects of adolescent emotional problem on activity at work day $(-0.01)$ and on sports/recreational activity $(-0.01)$ via adult depression/anxiety were of smaller 
magnitude. There were inverse associations between adult depression/anxiety with sports/recreational activity $(-0.06)$ and activity at work $(-0.07)$. Lower educational level was associated with a higher level of activity at work (0.31) and a lower level of sports/ recreational activity $(-0.12)$.

Conclusions We found that the effect of childhood socio-economic adversity on adult physical activity was entirely mediated by educational attainment. The association between adolescent depression/anxiety and activity was entirely mediated by current mental health problems. These results highlight the importance of education in reducing the adverse effect of childhood socioeconomic conditions on adult physical activity. Furthermore, addressing current mental health status should be seen as a priority for policies aimed at physical activity in adulthood.

\section{PHYSICAL ACTIVITY ACROSS ADULTHOOD AND PHYSICAL CAPABILITY IN MID-LIFE: FINDINGS FROM A BRITISH BIRTH COHORT STUDY}

doi:10.1136/jech.2010.120956.70

R Cooper, G Mishra, D Kuh. MRC Unit for Lifelong Health and Ageing and Division of Population Health, University College London, London, UK

Background Maintaining high levels of physical capability with age is important given that low levels are associated with increased risk of losing independence, health problems and mortality. Studies of older people provide evidence to suggest that physical activity may be beneficial for the maintenance of physical capability however it is unclear whether the effects of physical activity accumulate over the lifecourse.

Objectives To test the associations between physical activity levels, assessed by self-report of participation in sports and recreational activities prospectively at three ages across adulthood (36, 43 and 53 years), and objective measures of physical capability at age 53 year; to examine whether any associations found are independent of physical activity levels at other ages and other potential confounders.

Design Prospective cohort study.

Setting England, Scotland and Wales.

Participants Approximately 2400 men and women from the MRC National Survey of Health and Development, followed up since birth in March 1946.

Main outcome measures Grip strength, standing balance and chair rise time assessed by nurses during home visits at age 53 year.

Results Physical activity levels at all three ages in adulthood were positively associated with chair rise and standing balance performance. These associations were maintained after adjustment for sex, height, weight and socio-economic position with those people who were categorised as being most active performing better in these two tests than people reporting no activity. In models which included physical activity at all three ages simultaneously, there was evidence of independent positive effects of participation in sports and recreational activities at all three ages on chair rise performance and at ages 43 and 53 years on standing balance performance. Differences in mean chair rise time $(1 /$ time $(s) \times 100)$ between the most active and least active groups were: (at age 53 year: $0.30(95 \%$ CI 0.14 to 0.46$)$; at ages 36 and 43 year: $0.36(0.18,0.54)$ ) after adjustment for activity levels at the other two ages and covariates. There was no evidence of associations between physical activity levels at any age and grip strength in women and in men only physical activity at age 53 year was associated with grip strength. Conclusions Evidence of independent effects of physical activity at different ages across adulthood on chair rise and standing balance performance in mid-life suggests that there are cumulative benefits of physical activity across adulthood for physical capability in midlife. Increased activity should therefore be promoted earlier in life. 071 DIFFERENT EFFECTS OF BODY MASS INDEX AND PHYSICAL
ACTIVITY ON THE RISK OF ANKLE, WRIST AND HIP FRACTURES IN POSTMENOPAUSAL WOMEN

doi:10.1136/jech.2010.120956.71

M E G Armstrong, E A Spencer, B J Cairns, V Beral, for the Million Women Study Collaborators. Cancer Research UK Epidemiology Unit, University of Oxford, Oxford, UK

Objective To compare the relationship between body mass index (BMI) and physical activity for the risk of ankle, wrist and hip fractures in a large prospective study of postmenopausal women in the UK.

Methods In 1996-2001, women recruited into the Million Women Study completed a self-administered questionnaire asking about body size, physical activity, disease history, and lifestyle and reproductive factors. Incident ankle, wrist and hip fractures were identified through self-report in a follow-up questionnaire completed an average of 3.2 years after recruitment. RRs and CIs for each fracture site in postmenopausal women by BMI and physical activity at recruitment were calculated using Cox regression models, adjusted for socio-economic status, smoking and other health and lifestyle factors.

Results Among 599550 postmenopausal women, 5114 reported an ankle fracture, 8565 reported a wrist fracture, and 754 reported a hip fracture during follow-up. When compared to lean women $\left(\mathrm{BMI}<22.5 \mathrm{~kg} / \mathrm{m}^{2}\right)$, obese women $\left(\mathrm{BMI} \geq 30 \mathrm{~kg} / \mathrm{m}^{2}\right)$ had an increased risk of ankle fracture (RR 2.47; 95\% CI 2.32 to to 2.63), but a decreased risk of wrist fracture (RR $0.68 ; 95 \%$ CI 0.64 to to 0.72 ) and hip fracture (RR $0.29 ; 95 \%$ CI 0.23 to to 0.37$)\left(\chi^{2}<0.001\right.$ for the effect of BMI for each fracture site). Physical activity had little influence on the risk of either ankle fracture or wrist fracture, but women who reported strenuous physical activities up to once per week or more often were at a lower risk of hip fracture than women who reported less frequent activity (RR 0.65 ; 95\% CI 0.56 to to $0.76)$.

Conclusions BMI and physical activity have different effects on the incidence of fracture at different sites. While obese women are at increased risk of ankle fracture they are at lower risk of wrist fracture and hip fracture. Physical activity has no marked influence on ankle and wrist fracture but is protective against hip fracture.

\section{Adolescence}

072 UNDERSTANDING SOCIAL AND CULTURAL INFLUENCES ON THE RELATIONSHIPS AND SEXUAL EXPERIENCES OF YOUNG BRITISH PAKISTANIS IN LONDON: IS THERE UNMET SEXUAL HEALTH NEED?

doi:10.1136/jech.2010.120956.72

${ }^{1} \mathrm{C}$ Griffiths, ${ }^{2} \mathrm{~A}$ Prost, ${ }^{1} \mathrm{G}$ J Hart. ${ }^{1}$ Centre for Sexual Health and HIV Research, Department of Infection and Population Health, University College London, London, UK ${ }^{2}$ Centre for International Health and Development, University College London, London, UK

Background and aim Health services should take account of cultural and faith diversity. Pakistanis are the UKs second largest ethnic group and one of the largest Muslim communities. However, relative to other ethnic minority groups, there is a paucity of sexual health research among this group. Using community-based qualitative research we explored the social and cultural influences on sexual attitudes and experiences of young Pakistanis in East London to determine whether there is unmet sexual health need and implications for service development.

Design Between June and September 2008, 30 in-depth one-to-one interviews (60-90 min) were conducted with young Pakistanis 